16. Smith, S.A., et al. 2006. Polyphosphate modulates blood coagulation and fibrinolysis. Proc. Natl. Acad. Sci. U. S. A. 103:903-908.

17. Guerrini, M., et al. 2008. Oversulfated chondroitin sulfate is a contaminant in heparin associated with adverse clinical events. Nat. Biotechnol. 26:669-675.

18. Kishimoto, T.K., et al. 2008. Contaminated heparin associated with adverse clinical events and activation of the contact system. N. Engl. J. Med. 358:2457-2467.

19. Shariat-Madar, Z., Mahdi, F., and Schmaier, A.H. 2004. Recombinant prolylcarboxpeptidase activates plasma prekallikrein. Blood. 103:4554-4561.

20. Rojkjaer, R., Hasan, A.A.K., Motta, G., Schousboe, I., and Schmaier, A.H. 1998. Factor XII does not initiate prekallikrein activation on endothelial cells. Thromb. Haemost. 80:74-81.

21. Mahdi, F., Shariat-Madar, Z., Figueroa, C.D., and Schmaier, A.H. 2002. Factor XII interacts with the multiprotein assembly of urokinase plasminogen activator, $\mathrm{gC} 1 \mathrm{qR}$, and cytokeratin 1 on endothelial cell membranes. Blood. 99:3585-3596.

22. Gordon, E.M., et al. 1996. Factor XII-induced mitogenesis is mediated via a distinct signal transduction pathway that activates a mitogenactivated protein kinase. Proc. Natl. Acad. Sci. U.S. A. 93:2174-2179.

23. Zito, F., et al. 2002. Association of the factor XII
$46 \mathrm{C}>\mathrm{T}$ polymorphism with risk of coronary heart disease in the WOSCOPS study. Atherosclerosis. 165:153-158.

24. Renne, T., et al. 2005. Defective thrombus formation in mice lacking coagulation factor XII. J. Exp. Med. 202:271-281.

25. Merkulov, S., et al. 2008. Deletion of murine kininogen gene 1 (mKng1) causes loss of plasma kininogen and delays thrombosis. Blood. 111:1274-1281.

26. Shariat-Madar, Z., et al. 2006. Bradykinin B2 receptor knockout mice are protected from thrombosis by increased nitric oxide and prostacyclin. Blood. 108:192-199.

\title{
Platelet adhesion: a game of catch and release
}

\author{
Robert K. Andrews ${ }^{1}$ and Michael C. Berndt ${ }^{2}$
}

1Department of Immunology, Alfred Medical Research \& Education Precinct (AMREP), Monash University, Melbourne, Victoria, Australia. ${ }^{2}$ College of Medicine and Health, University College Cork, Cork, Republic of Ireland.

\begin{abstract}
The interaction of circulating platelets with the vessel wall involves a process of cell catch and release, regulating cell rolling, skipping, or firm adhesion and leading to thrombus formation in flowing blood. In this regard, the interaction of platelet glycoprotein Ib $\alpha$ (GPIb $\alpha$ ) with its adhesive ligand, $\mathrm{vWF}$, is activated by shear force and critical for platelet adhesion to the vessel wall. In this issue of the JCI, Yago and colleagues show how gain-of-function mutations in the GPIb $\alpha$-binding vWF A1 domain disrupt intramolecular interactions within WT vWF A1 that regulate binding to GPIb $\alpha$ and flowenhanced platelet rolling and adhesion (see the related article beginning on page 3195). Together, these studies reveal molecular mechanisms regulating GPIb $\alpha$-vWF bond formation and platelet adhesion under shear stress.
\end{abstract}

One of nature's mightiest forces is reptilian in origin. The gecko's foothold has a force of approximately $10 \mathrm{~N} / \mathrm{cm}^{2}$, allowing the gecko to more than support its own weight under the force of gravity (1). The biomechanics of this superb adaptation for reversible weight-bearing attachment to smooth surfaces involves hair-like structures (seta) on the footpad that end in multiple spatula-shaped projections capable of bearing approximately $20 \mu \mathrm{N}$ per seta. Altering the 3D orientation of the seta with the surface (related to uncurling and peeling movements of the toe) can increase or decrease detachment forces (1).

No less remarkable in the mammalian world are structural adaptations at the molecular level controlling receptor-medi-

Nonstandard abbreviations used: ADAMTS 13 , a disintegrin and metalloproteinase with a thrombospondin type 1 motif-13; AFM, atomic force microscopy; GP, glycoprotein; vWD, von Willebrand disease.

Conflict of interest: The authors have declared that no conflict of interest exists.

Citation for this article: J. Clin. Invest. 118:3009-3011 (2008). doi:10.1172/JCI36883. ated cell adhesion, in particular cell attachment under hydrodynamic shear flow in the bloodstream (2-8). In the case of blood platelets, this involves the reversible interaction between platelet glycoprotein Ib $\alpha$ (GPIba; the major ligand-binding subunit of the GPIb-IX-V complex) and vWF associated with an injured or diseased vessel wall. Fluid shear rates in flowing blood may be minimal at the center of the vessel, but become increasingly pronounced approaching the vessel wall as a result of drag (9). Shear stress on an adherent cell (force per unit area) depends on cell size, fluid viscosity, and proximity to the wall. At the cellular level, this has profound effects on initial contact, translocation, and firm adhesion of platelets under shear conditions. As shear rates increase from low physiological rates (less than $600 \mathrm{~s}^{-1}$ ) to high physiological rates (up to about $1,500 \mathrm{~s}^{-1}$ ) to pathological rates (up to about $10,000 \mathrm{~s}^{-1}$, such as in a stenotic artery), contact adhesion of platelets is increasingly dependent on GPIb $\alpha / \mathrm{vWF}$, and elongated tether-like structures may form parallel to the direction of flow $(10,11)$.
To date, little is understood about the mechanical properties of individual GPIb $\alpha$ / vWF molecular bonds, or how congenital point mutations affecting GPIb $\alpha-v W F$ binding affinity determine the physical interaction. In their study in this issue of the $J C I$, Yago et al. use atomic force microscopy (AFM) and molecular dynamics simulations to show how orientation of the GPIb-binding A1 domain of vWF with GPIba, involving alignment of specific amino acid residues, may regulate catch/slip bonding and release of GPIb $\alpha$ as shear force increases (8).

\section{vWF}

vWF is a multifunctional adhesive glycoprotein stored in platelet $\alpha$-granules and Weibel-Palade bodies of endothelial cells. In its mature form, it consists of disulfide-linked, approximately $275-\mathrm{kDa}$ subunits forming multimers of at least 20,000 kDa (Figure 1). Each subunit is composed of conserved domains: D'-D3-A1-A2-A3-D4-B1-B2-B3C1-C2 (12). The A1 domain encompassing the disulfide bond at C1272-C1458 (C509-C695 in the mature sequence) contains the binding site for GPIb $\alpha$, heparin, and other binding partners. The vWF A3 domain binds collagen, while $\mathrm{C} 1$ contains an Arg-Gly-Asp (RGD) sequence that binds the platelet integrin $\alpha_{\mathrm{II}} \beta_{3}$. The GPIb-binding site on vWF A1 is cryptic under static conditions, but becomes competent to bind receptor when matrix associated, exposed to shear force, or activated by nonphysiological modulators such as the bacterial glycopeptide ristocetin or the snake toxin botrocetin $(12,13)$. Ultralarge vWF multimers expressed on activated endothelial cells 


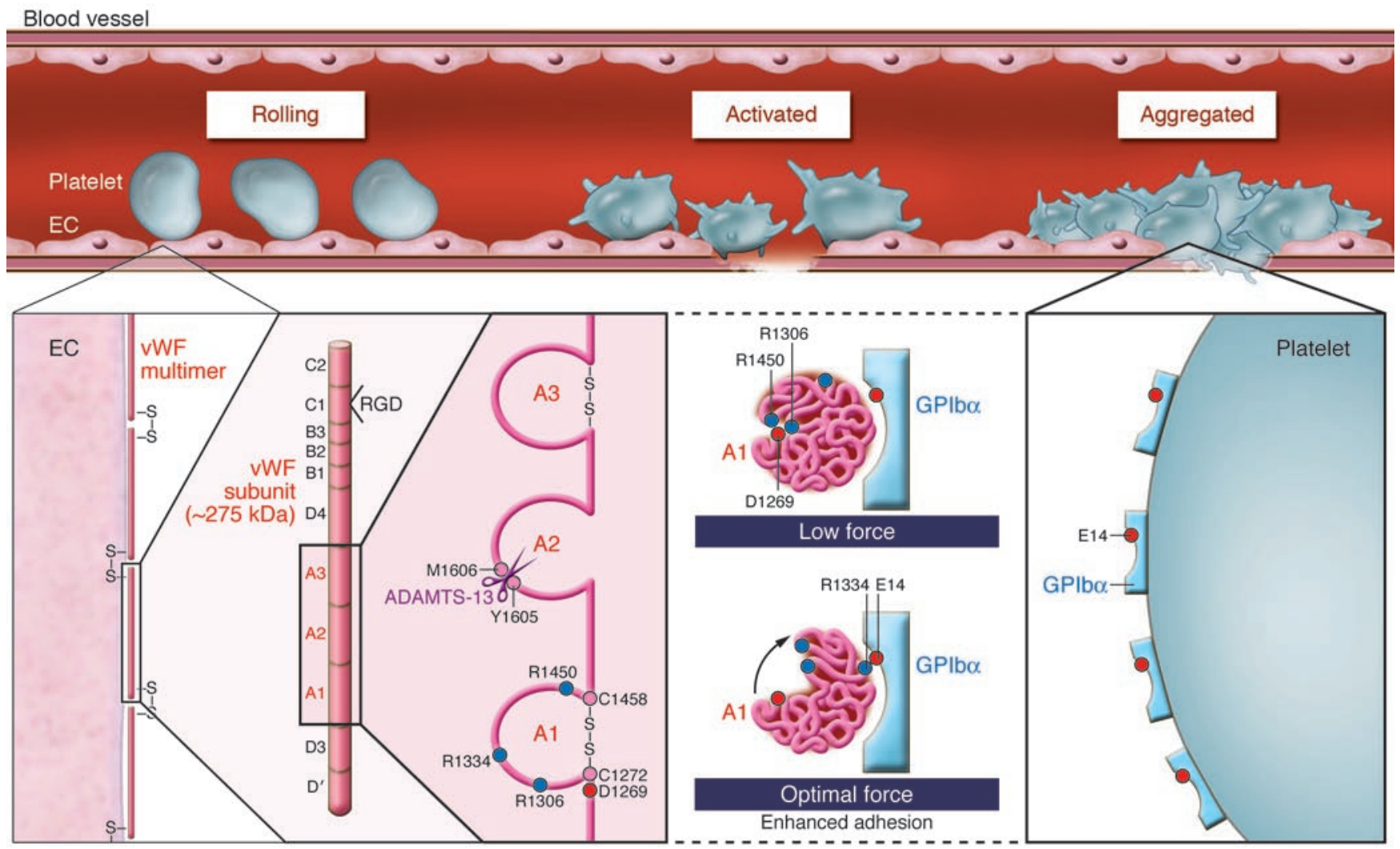

Figure 1

vWF-dependent platelet adhesion at high shear. GPIb-IX-V-dependent adhesion of human platelets to multimeric vWF on the vessel wall - initiating thrombus formation - involves GPIb $\alpha$ binding vWF A1, a conformationally activated domain of vWF. In their study in this issue of the $\mathrm{JCl}$, Yago et al. (8) show how shear force disrupts vWF A1 electrostatic interactions of D1269 with R1306 and R1450, reorientating vWF A1 relative to receptor and aligning R1334 of vWF to interact with E14 on GPIb $\alpha$, facilitating adhesion under flow (red and blue circles denote negatively and positively charged residues, respectively; see ref. 8 for structures). The authors show that vWD type 2B mutations (R1306Q or R1450E) constitutively disrupt interactions with D1269, enhancing binding to GPIb $\alpha$, as well as promoting ADAMTS-13-dependent cleavage within vWF A2. These findings explain how these vWD mutations lead to enhanced vWF binding to platelet GPIb $\alpha$ and depletion of vWF associated with type 2B vWD. In WT vWF, the shear-induced changes in the binding interaction between vWF and GPIb $\alpha$ show how platelet adhesion is enhanced under the influence of shear force and how thrombus formation can be initiated at the vessel wall in flowing blood.

are highly prothrombotic and bind platelets with high avidity. Under shear stress, the metalloproteinase a disintegrin and metalloproteinase with a thrombospondin type 1 motif-13 (ADAMTS-13) cleaves within the A2 domain, between Y1605 and M1606, decreasing vWF size and downregulating GPIb-dependent platelet adhesion.

\section{Platelet GPIb-IX-V}

Thrombus formation requires the initial contact of circulating platelets with the vessel wall, triggering platelet activation, shape change, and spreading as well as secretion of agonists such as ADP, leading to activation of $\alpha_{\mathrm{IIb}} \beta_{3}$ that binds vWF or fibrinogen and mediates platelet aggregation. Platelet GPIb $\alpha$ (of the GPIb-IX-V complex), which binds vWF, and the collagen receptor GPVI/FcR $\gamma$ form an adhesion-signaling complex unique to platelets and critical for thrombus formation at high physiological or pathological shear rates $(12,13)$. The vWF-binding domain of GPIb $\alpha-\mathrm{H} 1-\mathrm{E} 282$, which is elevated from the plasma membrane by a mucin stalk consists of an $\mathrm{N}$-terminal disulfide-looped capping sequence (residues 1-35), a leucinerich repeat sequence (residues 36-200), a C-terminal disulfide-looped flanking sequence (residues 201-268), and an anionic/sulfated sequence (residues 269-282) (12-14). Cocrystal structures show major contact points for vWF A1 clustered mainly within the $\mathrm{N}$-terminal capping and C-terminal flanking sequences and binding interfaces of electrostatic complementarity (14-16). The domain of GPIb $\alpha$ situated between residues 1 and 282 also contains binding sites for other adhesive ligands (e.g., thrombospondin), counter-receptors (e.g., P-selectin and the leukocyte integrin
$\alpha_{M} \beta_{2}$ ), and coagulation Factors XI and XII, kininogen, and thrombin (12).

\section{Bonding between vWF A1 and GPIb $\alpha$}

The AFM method Yago et al. describe in their current study (8) used a cantilever coated with GPIb $\alpha$ ectodomain (i.e., glycocalicin) or albumin-coated control, which was placed in contact with a petri dish surface coated with WT or mutant vWF A1 domain. It was left approximately $4 \mathrm{~nm}$ above the surface for a second to allow the bond to form; the probe was then retracted at a preset force, allowing measurement of whether a bond formed and how long it lasted, i.e., bond lifetime (8). Effects of shear on the GPIb $\alpha$-vWF interaction were also measured by passing platelets or GPIb-coated microspheres over immobilized vWF A1. Analysis of the WT vWF A1GPIb $\alpha$ interaction revealed that binding was biphasic, consistent with prolonged (catch) 
and shortened (slip) lifetime bonds. Catch and slip describe bonds under the influence of force: for catch bonds, force may enhance the binding interaction to prolong bond lifetime, for instance by conformational deformation; for slip bonds, force lowers the energy barrier between bound and free states, shortening bond lifetime (7). In their experiments, Yago et al. observed an upward trend with increasing force to an optimal level (catch bonds); as force further increased, bond lifetimes decreased (slip bonds) (8). This behavior of individual GPIb $\alpha$-vWF bonds under shear correlated with adhesion of platelets or beads to vWF under flow, where increasing shear forces initially decreased rolling velocity, transitioning to increased rolling velocity as the shear flow further increased. Breaking of the GPIb $\alpha-v W F$ bond was necessary for the rolling platelet to move a step forward, meaning that longer bond lifetimes decrease rolling velocity. That is, the type (catch/ slip) and strength (regulating attachment/ detachment) of the receptor/ligand interaction enabled the circulating cell to roll, skip, adhere, or detach depending on shear force.

\section{Other receptor/ligand interactions}

Bond strengths and dynamics have previously been analyzed for other adhesion receptors, revealing that multiphase bond formation is common. Cadherins, which form homotypic bonds, may initially form rapid, weak interactions followed by a lag and transition to a second, stronger binding state with a slow dissociation rate (2). Rupture forces for $\alpha_{\mathrm{IIb}} \beta_{3}$-fibrinogen complexes show increased affinity of activated conformers of $\alpha_{\mathrm{II}} \beta_{3}$ and 2 activation states $(3,4)$. However, like platelet GPIb $\alpha$-vWF adhesion, selectin-mediated interactions controlling leukocyte adhesion to the vessel wall (e.g., P-selectin-binding or L-selectin-binding carbohydrate ligands) are enhanced under flow conditions and involve analogous catch/slip interactions (5-7).

\section{Gain-of-function mutations and von Willebrand disease}

In human von Willebrand disease (vWD), vWF may be depleted (type 1), qualitatively abnormal (type $2 \mathrm{~A}$ or $2 \mathrm{~B}$ ), or virtually absent (type 3); type 2B vWD mutations are clustered within the vWF A1 domain and cause enhanced affinity for vWF binding to platelet GPIb $\alpha$ (11). Yago et al. also analyzed the effects of gain-of-function mutations R1306Q and R1450E in the vWF A1 domain on GPIb $\alpha$ bond formation as well as the relative importance of catch/slip bonding in GPIb $\alpha$-vWF-dependent adhesion
(8). Compared with WT, mutant vWF A1 containing R1306Q or R1450E overcame the dependence on shear for enhanced binding, eliminating catch bonds by prolonging lifetimes at low forces, and increasing rolling of platelets or beads as shear flow increased. Based on molecular dynamics simulations (simulating application of force to structures determined under static conditions), Yago et al. suggest that with WT vWF A1-GPIb $\alpha$, increasing force ruptures an intramolecular salt bridge connecting D1269 with R1306 or R1450, reorientating vWF A1 relative to receptor $\left(11^{\circ}\right.$ angular rotation $)$ and facilitating realignment of $\mathrm{R} 1334 \mathrm{of} \mathrm{VWF}$ to interact with E14 of GPIb $\alpha$, prolonging the bond lifetime (i.e., transition from slip to catch bonding). In comparison, R1306Q or R1450E mutations constitutively disrupt the bond with D1269, enhancing binding to GPIb $\alpha$ involving R1334 or E14 in the absence of shear. R1306Q or R1450E mutations in vWF A1 or A1A2A 3 constructs not only enhanced binding to GPIb $\alpha$, but also promoted ADAMTS-13-mediated cleavage within vWF A2. Together, these results account for vWF deficiency associated with these type $2 B$ mutations (although not necessarily all type 2B mutations, some of which do not involve charged amino acids). The results also show how electrostatic interactions - intramolecular bridging of D1269 and R1306 or R1450 and complementary residues of vWF (R1334) and GPIba (E14) - regulate GPIb $\alpha-v W F$ binding. Other evidence is consistent with the importance of electrostatic interactions between vWF and GPIb $\alpha$ (12). A negatively charged patch centered on D63 within leucine-rich repeats 2-4 (L60-E128) of GPIb $\alpha$ becomes increasingly important for GPIb-dependent adhesion to vWF as the shear rate increases (17); L60-E128 makes minimal direct contact with vWF A1 under static conditions, which suggests that shear-dependent conformational changes may occur in GPIba. Furthermore, mutation of K1362A within the A1 domain of mouse vWF ablates GPIb-dependent platelet binding in vivo, with increased bleeding and defective thrombus formation (18).

\section{Conclusions}

The present findings provide an understanding of how vWD mutations can affect vWF activity and how the interaction of vWF with GPIb $\alpha$ can be regulated under shear (8). The technology now available for investigating single bond strength and lifetime, together with whole-cell imaging under flow conditions, suggests ways of exploring therapeutic approaches based on disrupting adhesion kinetics, potentially attenuating GPIb-vWF-dependent thrombosis at pathological shear rates.

Address correspondence to:MichaelC.Berndt, College of Medicine and Health, Brookfield Health Sciences Complex, University College Cork, Western Road, Cork, Republic of Ireland. Phone: 353-21-4901616; Fax: 35321-4901549; E-mail: M.Berndt@ucc.ie.

1. Autumn, K., et al. 2000. Adhesive force of a single gecko foot-hair. Nature. 405:681-685.

2. Leckband, D. 2008. Beyond structure: mechanism and dynamics of intercellular adhesion. Biochem. Soc. Trans. 36:213-220.

3. Litvinov, R.I., et al. Functional and structural correlations of individual $\alpha \operatorname{IIb} \beta 3$ molecules. 2004. Blood. 104:3979-3985.

4. Litvinov, R.I., Bennett, J.S., Weisel, J.W., and Shuman, H. 2005. Multi-step fibrinogen binding to the integrin $\alpha \operatorname{IIb} \beta 3$ detected using force spectroscopy. Biophys. J. 89:2824-2834.

5. Edmondson, K.E., Denney, W.S., and Diamond, S.L. 2005. Neutrophil-bead collision assay: pharmacologically induced changes in membrane mechanics regulate the PSGL-1/P-selectin adhesion lifetime. Biophys. J. 89:3603-3614.

6. Lou, J., et al. 2006. Flow-enhanced adhesion regulated by a selectin interdomain hinge. J. Cell Biol. 174:1107-1117.

7. Marshall, B.T., et al. 2003. Direct observation of catch bonds involving cell-adhesion molecules. Nature. 423:190-193.

8. Yago, T., et al. 2008. Platelet glycoprotein Ib $\alpha$ forms catch bonds with human WT vWF but not with type $2 \mathrm{~B}$ von Willebrand disease vWF. J. Clin. Invest. 118:3195-3207.

9. Kroll, M.H., Hellums, J.D., McIntire, L.V., Schafer, A.I., and Moake, J.L. 1996. Platelets and shear stress. Blood. 88:1525-1541.

10. Jackson, S.P. 2007. The growing complexity of platelet aggregation. Blood. 109:5087-5095.

11. Maxwell, M.J., et al. 2007. Identification of a 2stage platelet aggregation process mediating shear-dependent thrombus formation. Blood. 109:566-576

12. Andrews, R.K., López, J.A., and Berndt, M.C. 1997. Molecular mechanisms of platelet adhesion and activation. Int. J. Biochem. Cell Biol. 29:91-105.

13. Andrews, R.K., López, J.A., and Berndt, M.C. 2006. The glycoprotein Ib-IX-V complex. In Platelets. 2nd edition. A.D. Michaelson, editor. Academic Press. San Diego, California, USA. 145-163.

14. Andrews, R.K., Gardiner, E.E., Shen, Y., Whisstock, J.C., and Berndt, M.C. 2003. Molecules in focus: Glycoprotein Ib-IX-V. Int. J. Biochem. Cell Biol. 35:1170-1174.

15. Huizinga, E.G., et al. 2002. Structures of glycoprotein Ib $\alpha$ and its complex with von Willebrand factor A1 domain. Science. 297:1176-1179.

16. Dumas, J.J., et al. 2004. Crystal structure of the wild-type von Willebrand factor A1-glycoprotein Ib $\alpha$ complex reveals conformation differences with a complex bearing von Willebrand disease mutations. J. Biol. Chem. 279:23327-23334.

17. Shen, Y., et al. 2006. Leucine-rich repeats 2-4 (Leu60-Glu128) of platelet glycoprotein Ib $\alpha$ regulate shear-dependent cell adhesion to von Willebrand factor. J. Biol. Chem. 281:26419-26423.

18. Marx, I., et al. 2008. Altered thrombus formation in von Willebrand factor-deficient mice expressing von Willebrand factor variants with defective binding to collagen or GPIIbIIIa. Blood. 112:603-609. 